\title{
Comparison of bone marrow-vs. adipose tissue-derived mesenchymal stem cells for attenuating liver fibrosis
}

\author{
TIANPAO HAO ${ }^{1 *}$, JINGFENG CHEN $^{2 *}$, SHAOCE ZHI ${ }^{1}$, QIYU ZHANG ${ }^{1}$, GANG CHEN $^{1}$ and FUXIANG YU ${ }^{1}$ \\ ${ }^{1}$ Department of Hepatobiliary and Pancreatic Surgery, The First Affiliated Hospital, Wenzhou Medical University, \\ Wenzhou, Zhejiang 325000; ${ }^{2}$ Department of Anorectal Surgery, The Sixth Affiliated Hospital of Wenzhou Medical University, \\ Lishui, Zhejiang 325000, P.R. China
}

Received February 12, 2017; Accepted August 4, 2017

DOI: 10.3892/etm.2017.5333

\begin{abstract}
Mesenchymal stem cell (MSC) therapy has emerged as a potential novel method of treating liver fibrosis. To date, bone marrow-derived MSCs (BM-MSCs) and adipose tissue-derived MSCs (AD-MSCs) have not been analyzed with respect to their ability to combat liver fibrosis. The present study aimed to compare the capabilities of BM-MSCs and AD-MSCs in the treatment of liver fibrosis. BM-MSCs and AD-MSCs were taken from male Sprague-Dawley rats and cultured. Hepatic stellate cells (HSCs) were co-cultured with either BM-MSCs or AD-MSCs, and the effects of BM-MSCs or AD-MSCs on the proliferation, activation and apoptosis of HSCs were determined. The secretion of a selected group of cytokines by BM-MSCs and AD-MSCs was measured using enzyme-linked immunosorbent assays. Using a $\mathrm{CCl}_{4}$-induced liver fibrosis animal model, the anti-inflammatory and anti-fibrotic effects of BM-MSCs or AD-MSCs against liver fibrosis in vivo were evaluated. The morphological examination and analysis of specific surface markers confirmed the successful preparation of BM-MSCs and AD-MSCs. Furthermore, the proliferation, activation and apoptosis of HSCs were significantly inhibited by BM-MSCs and AD-MSCs, with statistically greater reductions achieved by AD-MSCs compared with BM-MSCs. Direct comparison of the secretion of selected cytokines by BM-MSCs and AD-MSCs revealed that significantly higher levels of nerve growth factor and transforming grow th factor- $\beta 1$ were secreted in the AD-MSC culture medium, whereas levels of vascular endothelial growth factor and interleukin-10 did not differ significantly between AD-MSCs and BM-MSCs.
\end{abstract}

Correspondence to: Dr Fuxiang Yu or Dr Gang Chen, Department of Hepatobiliary and Pancreatic Surgery, The First Affiliated Hospital, Wenzhou Medical University, 233 Wenrui Avenue, Wenzhou, Zhejiang 325000, P.R. China

E-mail: yfxbmu@163.com

E-mail: cg_2188@163.com

${ }^{*}$ Contributed equally

Key words: bone marrow-derived mesenchymal stem cells, adipose tissue-derived mesenchymal stem cells, cell therapy, hepatic stellate cells, liver fibrosis
In vivo studies using a $\mathrm{CCl}_{4}$-induced liver fibrosis model demonstrated that inflammatory activity and fibrosis staging scores were significantly lower in the MSC-treated groups compared with controls. Although AD-MSCs improved anti-inflammatory and anti-fibrotic effects compared with BM-MSCs, these differences were not significant. Thus, the current study demonstrated that BM-MSCs and AD-MSCs are similarly effective at attenuating liver fibrosis by inhibiting the activation and proliferation of HSCs, as well as promoting the apoptosis of HSCs.

\section{Introduction}

Liver fibrosis is a common consequence of chronic liver diseases, including chronic hepatitis B virus infection, chronic hepatitis $\mathrm{C}$ virus infection, alcoholic liver disease and non-alcoholic fatty liver disease (1-4). During liver fibrogenesis, hepatic stellate cells (HSCs) are activated, there is an excessive accumulation of extracellular matrix in the liver and there is an increased inflammatory response (5). If left untreated or managed inappropriately, liver fibrosis may rapidly progress to advanced liver fibrosis or cirrhosis, which may result in patients requiring an orthotopic liver transplantation (1-3). Indeed, liver transplantation is currently the only available treatment option for patients diagnosed with the end-stage liver fibrosis or cirrhosis. However, a number of disadvantages, including a shortage of liver transplant donors, a high cost and transplant rejection following liver transplantation, have largely limited its clinical application. Therefore, novel therapeutic approaches are required for the treatment of liver fibrosis and to improve care for such patients.

Mesenchymal stem cell (MSC) transplantation (6-10) has emerged as an alternative and novel method of treating liver fibrosis regardless of the underlying cause (11). MSCs can be isolated from a wide range of tissue sources, including bone marrow, adipose tissue, umbilical cord/cord blood and dental pulp $(12,13)$. Of these MSCs, adipose tissue-derived MSCs (AD-MSCs) and bone marrow-derived MSCs (BM-MSCs) are the most easily accessible and have been extensively studied. Previous studies have demonstrated that MSCs possess immunomodulatory properties, the ability to differentiate into hepatocytes to replace damaged hepatocytes, and the capacity to suppress HSC activation and promote HSC 
apoptosis $(14,15)$. Several studies, including one by the current authors (16), revealed that BM-MSCs and AD-MSCs are capable of attenuating liver fibrosis using multiple molecular mechanisms by which HSC activation is inhibited, activated HSC proliferation is reduced, the inflammatory response is weakened and hepatocyte regeneration is enhanced (15-19). Indeed, AD-MSCs and BM-MSCs exhibit similar surface molecular markers and differentiation abilities; however, AD-MSCs are easier to obtain and have higher proliferation rates than $\mathrm{BM}-\mathrm{MSCs}$ (16).

To the best of our knowledge, AD-MSCs and BM-MSCs have not been compared with regards to their efficacy in the treatment of liver fibrosis. It remains unclear whether AD-MSCs and BM-MSCs exhibit equal anti-liver fibrosis and anti-inflammatory capabilities. The present study expanded upon the results of previous studies $(16,20)$ and performed comparative and comprehensive studies into the effects of BM-MSCs and AD-MSCs on the activation, proliferation and apoptosis of HSCs in a cell co-culture system, as well as their anti-inflammatory and anti-fibrotic properties in a $\mathrm{CCl}_{4}$-induced rat liver fibrosis model. Furthermore, the secretion of a select group of cytokines by BM-MSCs and AD-MSCs into the culture medium of a co-culture system was analyzed.

\section{Materials and methods}

Animals. A total of 30 Sprague-Dawley (SD) rats, aged 6 weeks old and weighing 200-250 g were obtained from the Animal Experimental Center of Wenzhou Medical College (Wenzhou, China). Of these, 5 rats were kept for one week until isolation of BM-MSCs and AD-MSCs, whereas 24 were also acclimatized for one week before they were used for induction of the $\mathrm{CCl}_{4}$-induced liver fibrotic rat model. Rats were maintained under conditions included in the animal care and research protocol, which was approved by the Animal Care and Use Committee of Wenzhou Medical College. The current study complied with the guidelines for the Care and Use of Laboratory Animals by the National Institutes of Health as described previously (16).

Culture of buffalo rat liver (BRL) cells and rat HSCs. The BRL-3A cell line was provided by the Experimental Surgical Center of Wenzhou Medical College (Wenzhou, China) and originally purchased from Procell Life Science Co. Ltd (Wuhan, China). BRL cells were incubated in Dulbecco's modified Eagle medium (DMEM; HyClone; GE Healthcare, Chicago, IL, USA) supplemented with $10 \%$ fetal bovine serum (FBS; Gibco; Thermo Fisher Scientific, Inc., Waltham, MA, USA) in a humidified atmosphere with $5 \% \mathrm{CO}_{2}$ at $37^{\circ} \mathrm{C}$. Rat primary HSCs were isolated from a male SD rat following a previously described protocol (16), and cultured in DMEM supplemented with $10 \% \mathrm{FBS}$ at $37^{\circ} \mathrm{C}$ in $5 \% \mathrm{CO}_{2}$ for $\sim 5-7$ days.

Preparation and culture of BM-MSCs and AD-MSCs. Fresh bone marrow and subcutaneous adipose tissues were obtained from male SD rats and used to isolate BM-MSCs and AD-MSCs. To prepare BM-MSCs, each end of the femur and tibia was cut to expose the marrow cavity, which was then washed with PBS at least three times. Fresh bone marrow was collected and centrifuged at $200 \mathrm{x}$ g at room temperature (RT) for $10 \mathrm{~min}$. Pelleted cells were resuspended in DMEM supplemented with $10 \%$ FBS and centrifuged at $200 \mathrm{x} g$ at RT for $10 \mathrm{~min}$. AD-MSCs were prepared by enzymatic digestion, as reported previously (16). Briefly, $1-2 \mathrm{mg}$ fresh adipose tissue was harvested from a male SD rat and washed with PBS. Tissues were digested with $1 \mathrm{mg} / \mathrm{ml}$ collagenase type IV (Sigma-Aldrich; Merck KGaA, Darmstadt, Germany) at $37^{\circ} \mathrm{C}$ for $1 \mathrm{~h}$. The suspension was then centrifuged at $200 \mathrm{x}$ g at RT for $10 \mathrm{~min}$. Pellets were subsequently resuspended in DMEM supplemented with $10 \%$ FBS and cultured in a humidified atmosphere with $5 \% \mathrm{CO}_{2}$ at $37^{\circ} \mathrm{C}$. The culture medium was changed every 2-3 days and the cells were passaged at $80 \%$ confluence. At passages 3-5, BM-MSCs and AD-MSCs were subjected to analysis of specific surface markers including cluster of differentiation (CD) 90, CD29 and CD45 by flow cytometry, as previously described (16). The proliferation and purity of the two types of MSCs were examined according to the instructions in the Trypan blue staining kit (cat. no. C0011-1; Beyotime Institute of Biotechnology, Haimen, China).

Co-culture of MSCs and HSCs. For co-culture of MSCs and HSCs, third-generation bone marrow- or AD-MSCs at a density of $3 \times 10^{4}$ cells/well were seeded onto the upper layer of Transwells (EMD Millipore, Billerica, MA, USA), while 3x10 HSCs/well were cultured in the basal compartment of a 6-well cell culture plate. $3 \times 10^{4}$ of BRLs/well were seeded instead of MSCs onto the upper layer of Transwells as a negative control and no cells were seeded onto the upper layer to serve as a blank control. All cells were maintained for $72 \mathrm{~h}$ in DMEM supplemented with $10 \%$ FBS in a humidified atmosphere with $5 \% \mathrm{CO}_{2}$ at $37^{\circ} \mathrm{C}$.

Cell proliferation assays. The cell counting kit-8 (CCK-8) (Dojindo Molecular Technologies, Inc. Kumamoto, Japan) was used to determine cell proliferation. In brief, $3 \times 10^{4}$ cells/well of BM-MSCs or AD-MSCs were co-cultured with HSCs at $3 \times 10^{4}$ cells/well for $72 \mathrm{~h}$, while the same amount of BRLs/well were seeded instead of MSCs as negative control and no cells were seeded as a blank control. Similarly as described above, under co-culture of MSCs and HSCs, all cells were maintained for $72 \mathrm{~h}$ in DMEM supplemented with $10 \% \mathrm{FBS}$ in a humidified atmosphere with $5 \%$ of $\mathrm{CO}_{2}$ at $37^{\circ} \mathrm{C}$. A total of $80 \mu \mathrm{l}$ CCK-8 reagent was added to each well containing HSCs in the basal compartment of a 6-well cell culture plate. Cells were incubated for an additional $2 \mathrm{~h}$ and absorbance was measured using a microplate reader at a wavelength of $450 \mathrm{~nm}$.

Flow cytometric analysis of cells undergoing apoptosis. Following co-culture of HSCs with BM-MSCs or AD-MSCs for $72 \mathrm{~h}$, cells were harvested and collected. Cells were washed twice with PBS and resuspended in $450 \mu$ l binding buffer (BD Biosciences, Franklin Lakes, NJ, USA). Samples were then incubated with $5 \mu \mathrm{l}$ Annexin $\mathrm{V}$ fluorescein isothiocyanate in the Annexin V-FITC kit (BD Biosciences) according to the manufacturer's instructions for $15 \mathrm{~min}$ at $4^{\circ} \mathrm{C}$ and flow cytometric analysis was performed to examine the proportion of HSCs undergoing apoptosis using Cell Quest Pro version 5.1 from BD Biosciences. 
Characterization of BM-MSCs and AD-MSCs was performed by using flow cytometry, during which, the expression of specific surface markers CD90, CD29 and CD45 was measured in fluorescence intensity over respective isotype and unstained negative control $\mathrm{Ig}$.

Western blot analysis. Western blot analysis was performed to examine the expression of $\alpha$-smooth muscle actin ( $\alpha$-SMA), following a previously described protocol (20). In brief, total protein samples were prepared from HSCs following co-culture with either BM-MSCs or AD-MSCs for $72 \mathrm{~h}$, separated using SDS-PAGE and transferred onto immunoblot polyvinylidene fluoride membranes. Membranes were blocked and then incubated with goat anti-mouse $\alpha$-SMA monoclonal primary antibody (cat. no. ab5694; Abcam, Cambridge, UK) at 1:200 dilution or anti-GAPDH (cat. no. ab9485; Abcam) as internal control at $4^{\circ} \mathrm{C}$ overnight, followed by an incubation with the secondary antibody rabbit anti-goat HRP (IgG H\&R) (cat. no. ab6741; Abcam) at a dilution of 1:30,000 at room temperature for $1 \mathrm{~h}$. Specific bands were visualized after using a developing solution from Beyotime Institute of Biotechnology (cat. no. P0019) and analyzed with a Gel-Pro analyzer imaging system from Media Cybernetics, Inc. (Rockville, MD, USA). The intensity of the $\alpha$-SMA bands was normalized to that of GAPDH.

Animal model of liver fibrosis and transplantation of MSCs. A total of 24 male SD rats weighing 250-300 g were selected to establish the animal model of $\mathrm{CCl}_{4}$-induced liver fibrosis. Rats were subcutaneously injected with $1.5 \mathrm{ml} / \mathrm{kg} \mathrm{CCl}$ diluted $1: 1$ (v/v) in olive oil twice a week to induce liver fibrosis. The rats were randomly divided into four groups: BM-MSC treatment $(n=6)$ and AD-MSC treatment $(n=6)$ groups, a BRL treatment group acting as a negative control $(n=6)$ and a group treated with cell culture medium containing no cells acting as a blank control $(n=6)$. For transplantation of BM-MSCs or AD-MSCs into the $\mathrm{CCl}_{4}$-treated rat liver, rats were anesthetized and the abdomen was incised to identify the portal vein to the liver. Subsequently, $5 \times 10^{6}$ BM-MSCs or AD-MSCs suspended in $1.5 \mathrm{ml}$ PBS were injected through the portal vein once every 2 weeks. Rats in the control groups were injected with $1.5 \mathrm{ml}$ PBS alone. Following treatment of the rats for 4 weeks, they were sacrificed and used for subsequent experiments.

Immunohistochemical staining. Paraffin-embedded sections (4-mm in thickness) of the liver were prepared following a previously published protocol (16), dewaxed in xylene, hydrated with distilled water and incubated with $3 \% \mathrm{H}_{2} \mathrm{O}_{2}$ at room temperature for $30 \mathrm{~min}$. Following washing with 1X PBS, slides were blocked with $2 \%$ goat serum in $0.01 \mathrm{M}$ PBS containing $0.3 \%$ Triton X-100 (PBS-X) for 1 hat room temperature, and subsequently incubated with $\alpha$-SMA primary antibody (cat. no. 5694; Abcam) at a dilution of 1:200 at room temperature for $1 \mathrm{~h}$ and rinsed with $1 \mathrm{X} \mathrm{PBS}$ at room temperature. A working solution with secondary antibody rabbit anti-goat HRP (IgG H\&R; cat. no. ab6741; Abcam) was added at a dilution of 1:30,000 and incubated at room temperature for $30 \mathrm{~min}$ Subsequently, the slides were visualized using an Olympus microscope (Model IX71) with magnification, x200 following development with diaminobenzine, counterstaining with hematoxylin, dehydration and mounting on a neutral resin. Brown staining indicated $\alpha$-SMA expression. Inflammation and fibrosis scores were evaluated according to the Ishak modified histological activity index (21). Masson staining was performed using a Masson staining kit (cat. no. HT15; Sigma-Aldrich; Merck KGaA) according to the manufacturer's instructions.

Measurement of cytokine secretion by ELISA. BM-MSCs or AD-MSCs at a density of $2 \times 10^{5}$ cells/well were seeded onto 6-well plates. BRLs were seeded instead of MSCs to act as a negative control. After $72 \mathrm{~h}$ culture, the cell culture medium was collected to measure cytokine secretion using ELISA kits (R\&D Systems, Inc., Minneapolis, MN, USA) for vascular endothelial growth factor (VEGF) (cat. no. RRV00), interleukin-10 (IL-10) (cat. no. R1000), nerve growth factor (NGF) (cat. no. DY556) and transforming growth factor- $\beta 1$ (TGF- $\beta 1$ ) (cat. no. MB100B), following the manufacturer's protocol.

Determination of collagen content in rat liver tissue and serum. Collagen content in rat liver tissue was examined using a hydroxyproline determination kit (cat. no. KA4552; Abnova, Taipei, Taiwan) in which hydroxyproline served as a measure of collagen concentration. Briefly, $80 \mathrm{mg}$ rat liver tissue was homogenized and centrifuged at $100 \mathrm{x}$ g at $4^{\circ} \mathrm{C}$ for $60 \mathrm{sec}$, after which supernatants were collected for subsequent hydroxyproline measurement. Absorbance at $550 \mathrm{~nm}$ was determined with a spectrophotometer. collagen content within the liver tissue was calculated according to the Hydroxyproline Assay kit protocol provided by Abnova. Serum type III collagen were determined by ELISA using an ELISA kit from Kamiya Biomedical Company (Shanghai, China; cat. no. KT-11210), hyaluronic acid levels were measured with an ELISA kit from Wuhan Fine Biotech Ltd. (Wuhan, China; cat. no. EU2556).

Statistical analysis. The experiments conducted in the present study included triplicate samples for each treatment group. All statistical analyses were performed using SPSS version 13.0 (SPSS Inc., Chicago, IL, USA). All data are presented as the mean \pm standard deviation, and a comparison among multiple groups was performed using analysis of variance and Tukey's post-hoc tests. $\mathrm{P}<0.05$ was considered to indicate a statistically significant difference.

\section{Results}

Preparation and characterization of BM-MSCs and $A D$-MSCs. BM-MSCs and AD-MSCs were prepared from the freshly harvested bone marrow and adipose tissue of a male SD rat. Trypan blue staining revealed a high degree of proliferation (92-98\%) and purity (80-95\%) of the two types of MSCs (data not shown). After the cells were cultured for $48 \mathrm{~h}$, cell morphology was examined using an inverted microscope. The vast majority of the cells were adherent and had a spindle or trigonal shape and only a small number of suspended cells were visualized. After 3-5 days, the cells reached $\sim 80 \%$ confluence, at which time the detached cells were washed with PBS and passaged with a small number of the cells transferred into a new vessel. There were no marked differences between the morphology of BM-MSCs and 

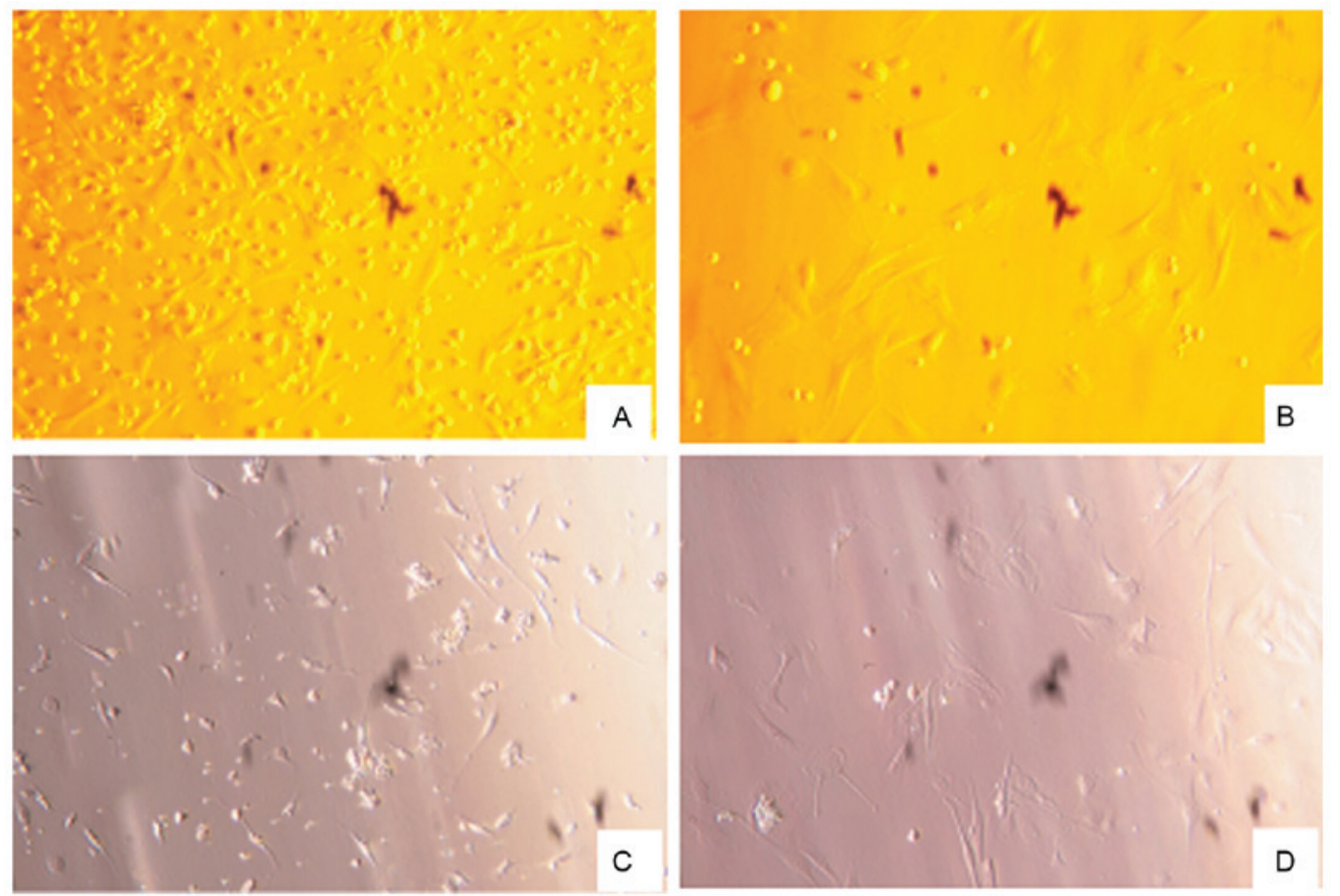

Figure 1. Morphology of rat BM-MSCs and AD-MSCs. BM-MSCs and AD-MSCs were isolated using bone marrow and adipose tissue freshly harvested from a male Sprague-Dawley rat. Magnification, x200. (A) Primary and (B) third-generation BM-MSCs; (C) primary and (D) third-generation AD-MSCs. BM-MSCs, bone marrow-derived mesenchymal stem cells; AD-MSCs, adipose tissue-derived mesenchymal stem cells.
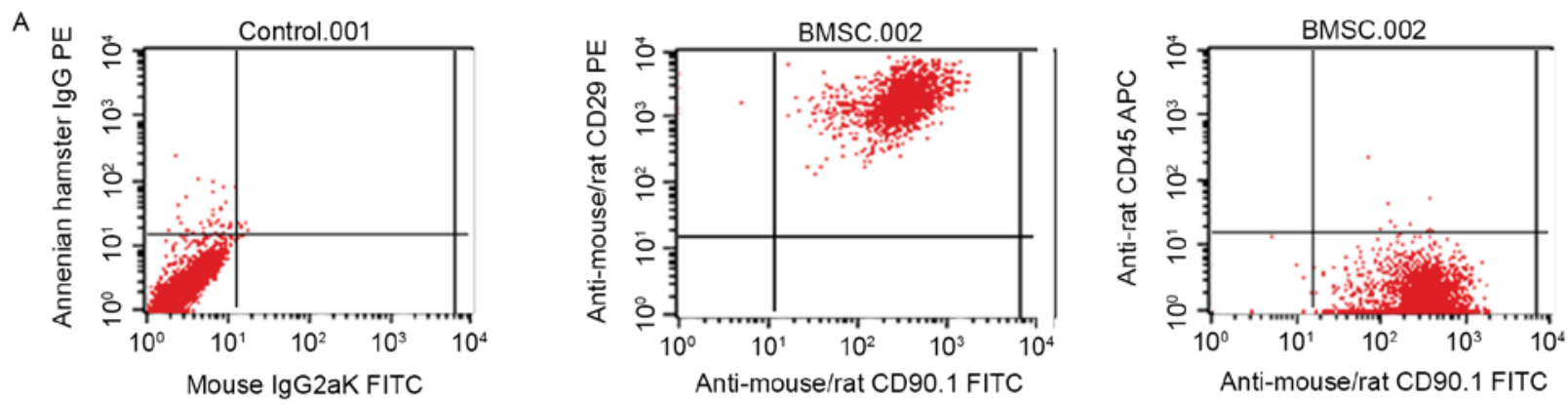

Primary MDSCs (CD90+: 99.0\%; CD29+: 98.5\%; CD45-: 2.1\%)

B

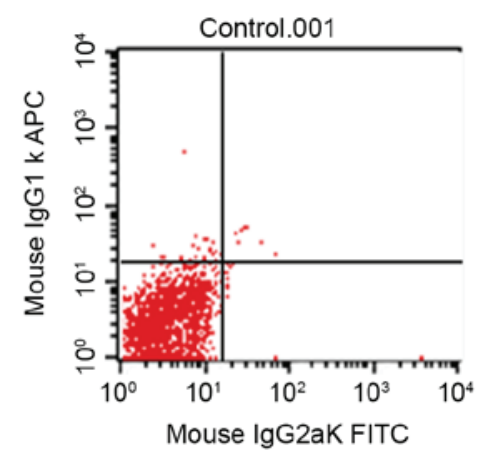

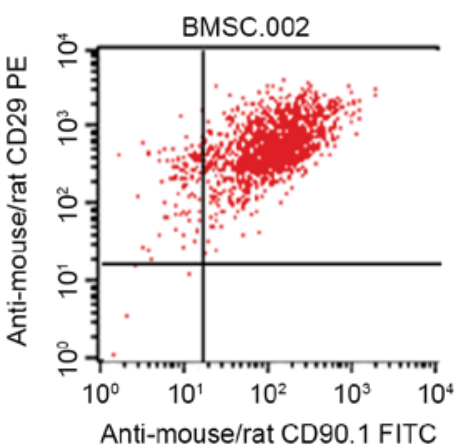

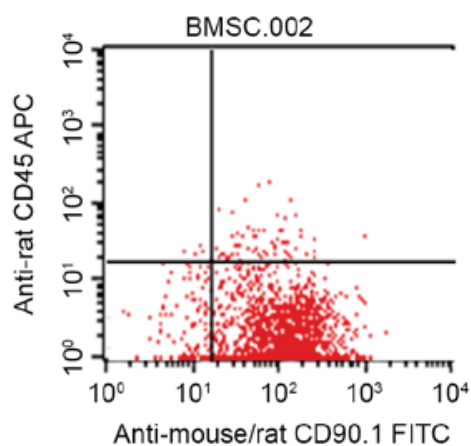

Anti-mouse/rat CD90.1 FITC

Primary ADSCs (CD90+: 93.4\%; CD29+: 99.7\%; CD45-: 3.8\%)

Figure 2. Characterization of BM-MSCs and AD-MSCs by flow cytometry. BM-MSCs and AD-MSCs were prepared from freshly harvested bone marrow and adipose tissues of a male Sprague-Dawley rat. The expression of specific surface markers was determined using flow cytometry. The expression of CD90, CD29 and CD45 was measured according to fluorescence intensity over respective isotype and unstained negative control Ig in (A) BM-MSCs and (B) AD-MSCs. BM-MSCs, bone marrow-derived mesenchymal stem cells; AD-MSCs, adipose tissue-derived mesenchymal stem cells; CD, cluster of differentiation; Ig, immunoglobulin; FITC, fluorescein isothiocyanate; PE, phycoerythrin; APC, allophycocyanin.

AD-MSCs (Fig. 1). Subsequently, the two types of stem cells were characterized based on the expression of their specific surface markers by fluorescence-activated cell sorting flow cytometry. As depicted in Fig. 2A and B, 99.0 and 98.5\% 
of BM-MSCs expressed CD90 and CD29, respectively, and 93.4 and $99.7 \%$ of AD-MSCs expressed CD90 and CD29, respectively, indicating that MSC surface markers were highly expressed by BM-MSCs and AD-MSCs. By contrast, the positive rates of the hematopoietic cell origin marker CD45 expression were low; only $2.1 \%$ of BM-MSCs and $3.8 \%$ of AD-MSCs expressed CD45 (Fig. 2). The morphological examination and the identification of MSC surface markers confirmed the successful preparation of BM-MSCs and AD-MSCs.

Effects of BM-MSCs and AD-MSCs on the activation, proliferation and apoptosis of HSCs in a co-culture system. HSC activation serves a pivotal role in the pathogenesis of liver fibrosis and $\alpha$-SMA is a classical molecular marker for activated HSCs (22); therefore, the effects of the two types of MSCs on the levels of $\alpha$-SMA were determined in the current study. Western blot analysis revealed that expression of $\alpha$-SMA was significantly reduced in HSCs co-cultured with either type of MSCs, compared with the expression in the control groups $(\mathrm{P}<0.05$; Fig. 3A). The effects of BM-MSCs and AD-MSCs on the expression of $\alpha$-SMA were compared and it was demonstrated that the AD-MSC co-culture group exhibited significantly lower $\alpha$-SMA expression $(\mathrm{P}<0.05$; Fig. $3 \mathrm{~A})$.

Subsequently, the effects of BM-MSCs and AD-MSCs on the proliferation and apoptosis of HSCs were compared after $72 \mathrm{~h}$ co-culture. Compared with that of the controls, the proliferation of HSCs was significantly inhibited by co-culture with either BM-MSCs or AD-MSCs $(\mathrm{P}<0.05)$, with a significantly greater reduction in HSC proliferation achieved by AD-MSCs than BM-MSCs ( $<<0.05$; Fig. 3B). Data demonstrated that the two types of MSCs promoted the apoptosis of HSCs, with a higher rate of apoptosis induced by AD-MSCs compared with BM-MSCs ( $\mathrm{P}<0.05$; Fig. 3C).

Comparison of cytokine secretion by BM-MSCs and $A D$-MSCs in co-culture with HSCs. The aforementioned observations indicate that BM-MSC and AD-MSCs were able to reduce liver fibrosis in the cell co-culture system. Since there was no direct cell-cell contact between HSCs and MSCs, the secretion of a select group of cytokines into the cell culture medium by MSCs was evaluated. It has been documented that VEGF, IL-10, NGF and TGF- $\beta 1$ are associated with the control of cellular functions $(18,19,23)$. As presented in Table I, compared with the control cells, each type of MSC secreted significantly more VEGF (BM-MSCs vs. BRLs: $\mathrm{P}<0.05$; AD-MSCs vs. $\mathrm{BRLs}, \mathrm{P}<0.05)$ and significantly less NGF (BM-MSCs vs. BRLs, $\mathrm{P}<0.05$; AD-MSCs vs. BRLs, $\mathrm{P}<0.05)$ and TGF- $\beta 1$ (BM-MSCs vs. BRLs, $\mathrm{P}<0.05$; AD-MSCs vs. BRLs, $\mathrm{P}<0.05)$ compared with the control. IL-10 levels did not differ between MSCs and the control. Furthermore, NGF and TGF- $\beta 1$ levels in the AD-MSC culture medium were significantly higher than those in the BM-MSC culture medium (AD-MSCs vs. BM-MSCs, $\mathrm{P}<0.05$ ), whereas levels of VEGF and IL-10 in the media of the two MSC types did not differ significantly (AD-MSCs vs. BM-MSCs: P>0.05).

Anti-inflammatory and anti-fibrotic effects of BM-MSC and $A D$-MSC transplantation in a rat model of $\mathrm{CCl}_{4}$-induced liver fibrosis. Prior to assessment of the anti-inflammatory and

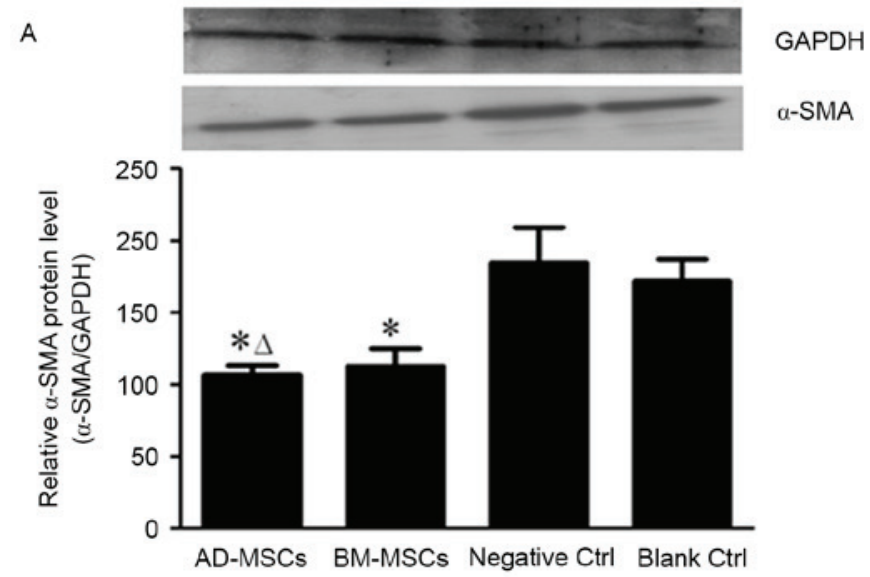

B

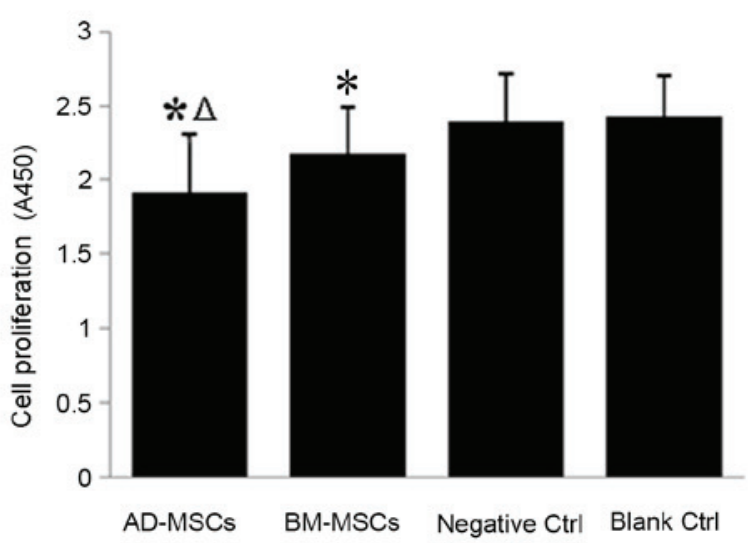

C

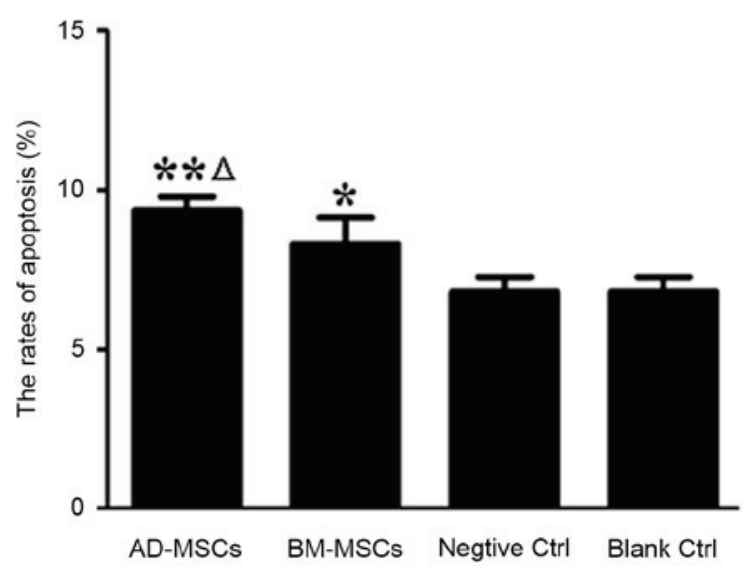

Figure 3. Effects of BM-MSCs and AD-MSCs on the activation, proliferation and apoptosis of HSCs in a co-culture system. HSCs were co-cultured with either BM-MSCs or AD-MSCs for $72 \mathrm{~h}$ and HSC activation, proliferation and apoptosis were examined. (A) Western blot analysis of $\alpha$-SMA expression in the control and MSC-treated groups. ${ }^{*} \mathrm{P}<0.05$ vs. control groups, ${ }^{\Delta} \mathrm{P}<0.05$ vs. BM-MSCs. (B) HSC proliferation in the control and MSC-treated groups. ${ }^{*} \mathrm{P}<0.05$ vs. control groups; ${ }^{\Delta} \mathrm{P}<0.05$ vs. BM-MSCs. (C) Apoptotic HSCs in the control and MSC-treated groups. BM-MSCs, bone marrow-derived mesenchymal stem cells; AD-MSCs, adipose tissue-derived mesenchymal stem cells; HSCs, hepatic stellate cells; $\alpha$-SMA, $\alpha$-smooth muscle actin. ${ }^{*} \mathrm{P}<0.05$ vs. control; ${ }^{* *} \mathrm{P}<0.05$ vs. control, ${ }^{\Delta} \mathrm{P}<0.05$ vs. BM-MSCs.

anti-fibrotic effects of BM-MSC and AD-MSC transplantation in rats, we validated the rat model of $\mathrm{CCl}_{4}$-induced liver fibrosis. Compared with the control group treated with vehicle olive oil only (Fig. 4A), rats exposed to $\mathrm{CCl}_{4}$ exhibited typical features of liver inflammation and fibrogenesis as revealed 
Table I. Levels of VEGF, IL-10, NGF and TGF- $\beta 1$ secreted into the cell culture medium of HSCs co-cultured with BRLs, BM-MSCs or AD-MSCs.

\begin{tabular}{llccc}
\hline Co-culture group & VEGF $(\mathrm{pg} / \mathrm{ml})$ & $\mathrm{IL}-10(\mathrm{pg} / \mathrm{ml})$ & NGF $(\mathrm{pg} / \mathrm{ml})$ & $\mathrm{TGF}-\beta 1(\mathrm{pg} / \mathrm{ml})$ \\
\hline BRLs & $21.08 \pm 5.15$ & $17.91 \pm 3.16$ & $10.13 \pm 1.52$ & $53.27 \pm 12.68$ \\
BM-MSCs & $80.33 \pm 14.48^{\mathrm{a}}$ & $18.12 \pm 1.53$ & $3.95 \pm 0.71^{\mathrm{a}}$ & $6.36 \pm 0.85^{\mathrm{a}}$ \\
AD-MSCs & $78.52 \pm 15.79^{\mathrm{a}}$ & $17.37 \pm 1.92$ & $7.46 \pm 0.54^{\mathrm{a}, \mathrm{b}}$ & $8.79 \pm 0.93^{\mathrm{a}, \mathrm{b}}$ \\
\hline
\end{tabular}

Data are presented as the mean \pm standard deviation. ${ }^{\mathrm{a}} \mathrm{P}<0.05 \mathrm{vs}$. BRLs as the control; ${ }^{\mathrm{b}} \mathrm{P}<0.05 \mathrm{vs}$. BM-MSCs. VEGF, vascular endothelial growth factor; IL-10, interleukin-10; NGF, nerve growth factor; TGF- $\beta 1$, transforming growth factor- $\beta 1$; HSCs, hepatic stellate cells; BRL, buffalo rat liver cells; BM-MSCs, bone marrow-derived mesenchymal stem cells; AD-MSCs, adipose tissue-derived mesenchymal stem cells.

by hematoxylin and eosin (Fig. 4B), and Masson staining (Fig. 4C). These features included an increase in the density of collagen fibers, markedly proliferated fibrous tissue around the portal areas, inflammatory cell filtration and the formation of lobules. Pathological examination and measurement of liver fibrotic marker expression in the liver and serum samples from the control groups and the groups treated with the two types of MSCs were performed. Immunocytochemical staining revealed decreased hepatic steatosis, a smaller amount of fake leaflets and thinner fibre cords in the MSC-treated groups. In addition, the formation of fibrosis in the periportal areas and hepatic parenchyma were reduced in the MSC-treated groups (Fig. 5A and B). By contrast, the following pathological changes occurred in the control groups: Marked hepatic steatosis, fibrogenesis in the periportal areas and hepatic parenchyma, wide and thick cords of collagen fibers and a large amount of fake leaflets (Fig. 5C and D).

Furthermore, liver inflammation and fibrosis staging scores were compared between the control and MSC-treated groups. In the MSC-treated groups, the inflammation activity (BM-MSCs: F=51.26, $\mathrm{P}<0.05$; AD-MSCs: $\mathrm{F}=46.73, \mathrm{P}<0.05$ ) and fibrosis staging scores (BM-MSCs: $\mathrm{F}=32.29, \mathrm{P}<0.05$; AD-MSCs: $F=40.94, \mathrm{P}<0.05)$ were significantly lower in the MSC-treated groups compared with the control groups. Although implantation of AD-MSCs resulted in a slight improvement in anti-inflammatory and anti-fibrotic effects compared with implantation of BM-MSCs, the differences between the two groups were not significant $(\mathrm{P}>0.05$; Table II).

Serum hyaluronic acid, collagen type III and intrahepatic hydroxyproline are well-documented markers of fibrosis and may indicate the degree of liver fibrosis (24). To further compare the protective effects of implantation of the two types of MSCs, inflammation and fibrosis were induced in rats by $\mathrm{CCl}_{4}$ treatment and ELISA was performed to measure the serum levels of hyaluronic acid, collagen type III and intrahepatic hydroxyproline. As depicted in Table III, the serum concentrations of hyaluronic acid, collagen type III and intrahepatic hydroxyproline were significantly lower in the two MSC-treated groups than in the control groups $(\mathrm{F}=73.51$, $\mathrm{P}<0.05$ for hyaluronic acid; $\mathrm{F}=76.19, \mathrm{P}<0.05$ for collagen type III; and $\mathrm{F}=60.37, \mathrm{P}<0.05$ for intrahepatic hydroxyproline). Although transplantation of AD-MSCs resulted in slightly decreased serum levels of the fibrotic markers, the differences in serum levels in the two types of MSCs were not significant (P>0.05; Table III).
Table II. Liver inflammation and fibrosis staging scores in the control, BM-MSC and AD-MSC transplanted groups.

\begin{tabular}{lcc}
\hline Group & $\begin{array}{c}\text { Inflammatory } \\
\text { activity }\end{array}$ & $\begin{array}{c}\text { Fibrosis } \\
\text { staging score }\end{array}$ \\
\hline Blank control & $13.78 \pm 2.53$ & $5.09 \pm 1.15$ \\
Negative control & $13.34 \pm 1.89$ & $4.95 \pm 1.22$ \\
BM-MSCs & $9.87 \pm 2.07^{\mathrm{a}}$ & $4.17 \pm 0.94^{\mathrm{a}}$ \\
AD-MSCs & $10.13 \pm 1.81^{\mathrm{a}}$ & $3.98 \pm 0.82^{\mathrm{a}}$ \\
\hline
\end{tabular}

Data are presented as the mean \pm standard deviation. Inflammation and fibrosis scores were evaluated according to the Ishak modified histological activity index (21). ${ }^{a} \mathrm{P}<0.05$ vs. controls. BM-MSCs, bone marrow-derived mesenchymal stem cells; AD-MSCs, adipose tissue-derived mesenchymal stem cells.

\section{Discussion}

The present study compared the ability of two types of MSCs; BM-MSCs and AD-MSCs, to attenuate liver fibrosis using an in vitro co-culture system and an in vivo rat model of liver fibrosis. The major observations were as follows: i) BM-MSCs and AD-MSCs significantly inhibited the proliferation and activation of HSCs, with a statistically greater effect achieved by AD-MSCs compared with BM-MSCs in a co-culture system; ii) comparison of secretion of four cytokines by BM-MSCs and AD-MSCs into the cell culture medium identified similar levels of VEGF and IL-10 secretion between the two groups but significantly higher levels of NGF and TGF- $\beta 1$ secretion by AD-MSCs compared with BM-MSCs; and iii) the inflammatory activity and fibrosis staging scores in a rat model of $\mathrm{CCl}_{4}$-induced liver fibrosis were significantly lower in the BM-MSC- and AD-MSC-treated groups than in the control groups. AD-MSC implantation induced slightly improved anti-inflammatory and anti-fibrotic effects compared with BM-SC implantation; however, this difference was not significant.

Over the past few decades, studies have demonstrated that MSCs are able to differentiate into hepatocytes, secrete a variety of cytokines to modulate the immune responses and indicated that they possess potent anti-oxidant properties. These capabilities allow MSCs to combat liver fibrosis regardless of etiological factors (25-28). Indeed, MSC therapy 
Table III. Serum levels of hyaluronic acid, collagen III and hydroxyproline in control, BM-MSC and AD-MSC transplanted groups.

\begin{tabular}{lccc}
\hline Group & Hyaluronic acid $(\mu \mathrm{g} / \mathrm{l})$ & Collagen III $(\mu \mathrm{g} / \mathrm{l})$ & Hydroxyproline $(\mu \mathrm{g} / \mathrm{l})$ \\
\hline Blank control & $287.5 \pm 26.7$ & $32.5 \pm 4.3$ & $473.9 \pm 63.7$ \\
Negative control & $282.3 \pm 18.7$ & $35.3 \pm 3.3$ & $458.4 \pm 38.1$ \\
BM-MSCs & $191.5 \pm 33.2^{\mathrm{a}}$ & $19.9 \pm 5.1^{\mathrm{a}}$ & $312.6 \pm 38.8^{\mathrm{a}}$ \\
AD-MSCs & $178.8 \pm 28.2^{\mathrm{a}}$ & $21.7 \pm 3.3^{\mathrm{a}}$ & $325.8 \pm 28.2^{\mathrm{a}}$ \\
\hline
\end{tabular}

Data are presented as the mean \pm standard deviation. Serum hyaluronic acid, collagen type III, and intrahepatic hydroxyproline concentrations in serum were measured by ELISA. ${ }^{a} \mathrm{P}<0.05$ vs. controls. BM-MSCs, bone marrow-derived mesenchymal stem cells; AD-MSCs, adipose tissue-derived mesenchymal stem cells.
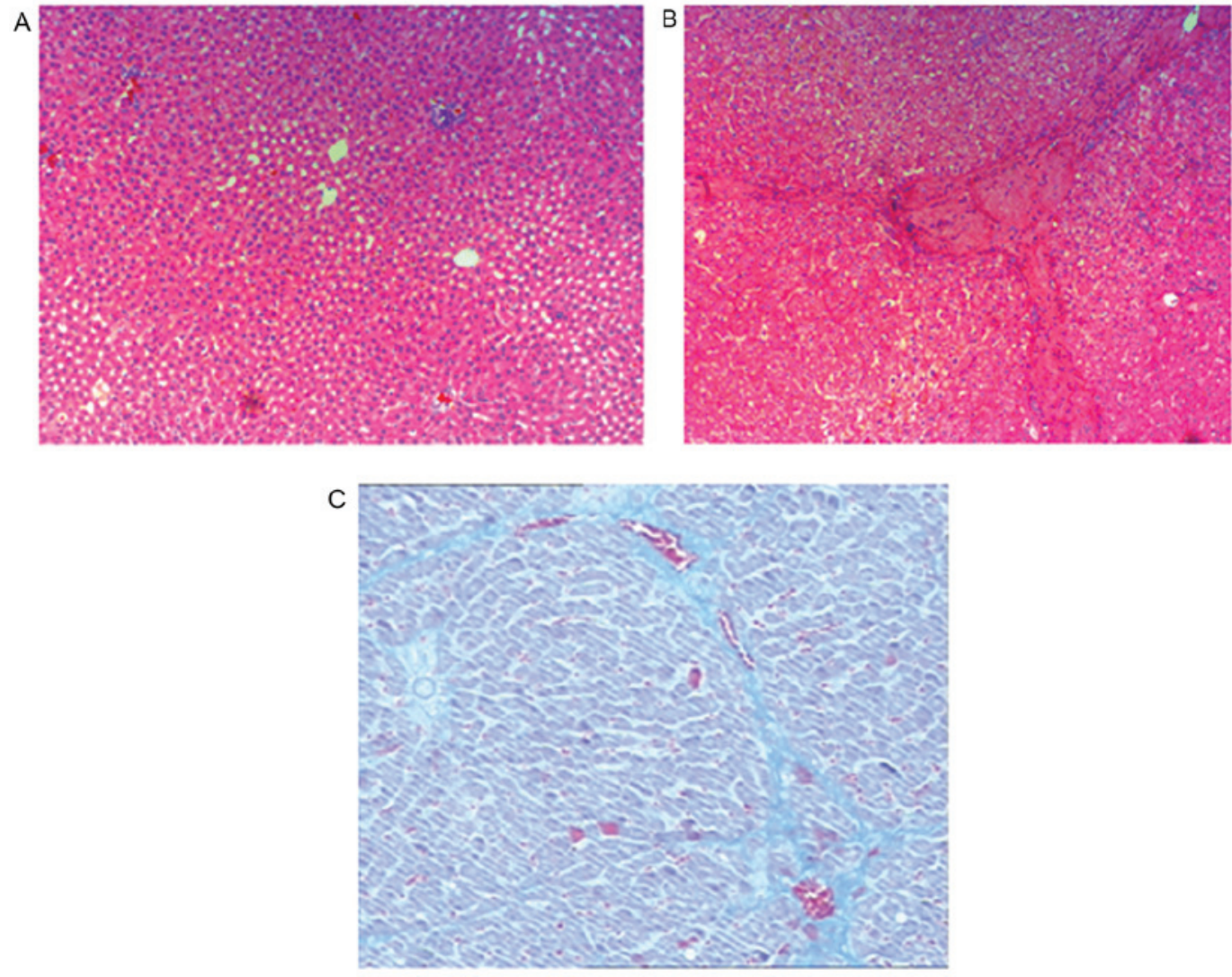

Figure 4. Establishment of a rat model of liver fibrosis induced by $\mathrm{CCl}_{4}$. Male Sprague Dawley rats were treated with $\mathrm{CCl}_{4}$ to produce a rat model of $\mathrm{CCl}_{4}$-induced liver fibrosis and establishment of liver fibrosis was determined by $\mathrm{H} \& \mathrm{E}$ or Masson staining. (A) $\mathrm{H} \& \mathrm{E}$ staining of the liver sections of the control rats treated with olive oil vehicle alone; (B) $\mathrm{H} \& \mathrm{E}$ staining of the liver sections of the rats treated with $\mathrm{CCl}_{4}$ diluted in olive oil and (C) Masson staining of the liver sections of the rats treated with $\mathrm{CCl}_{4}$ diluted in olive oil (magnification, x200). H\&E, hematoxylin and eosin.

has been widely studied and suggested as an alternative novel method of treating liver fibrosis (29-30). Furthermore, the development of MSC therapy may bring hope to patients with end-stage liver fibrosis on the waiting list for a liver transplant (30). MSCs can be derived from a wide range of tissue sources, including the bone marrow, adipose tissue, dental pulp and umbilical cord blood $(12,13)$. Furthermore, the differences and similarities of MSCs isolated from different sources and maintained in different culture conditions have been investigated $(12,13,16)$. The results of the present study indicate that BM-MSCs and AD-MSCs significantly inhibit the proliferation and activation of HSCs as well as promote apoptosis in HSCs compared with controls. These observations are consistent with those described in previous reports (30). The current study compared the effects of BM-MSCs and AD-MSCs on the cellular functions of HSCs and it was revealed that AD-MSCs were significantly more effective at inhibiting HSC proliferation and promoting HSC apoptosis than BM-MSCs. There was a similar effect on HSC activation. Further analysis of cytokine secretion by BM-MSCs and AD-MSCs in the co-culture system demonstrated that levels of NGF and TGF- $\beta 1$ were significantly greater in the AD-MSC culture medium than in the BM-MSC culture medium, whereas no significant differences were detected in VEGF 

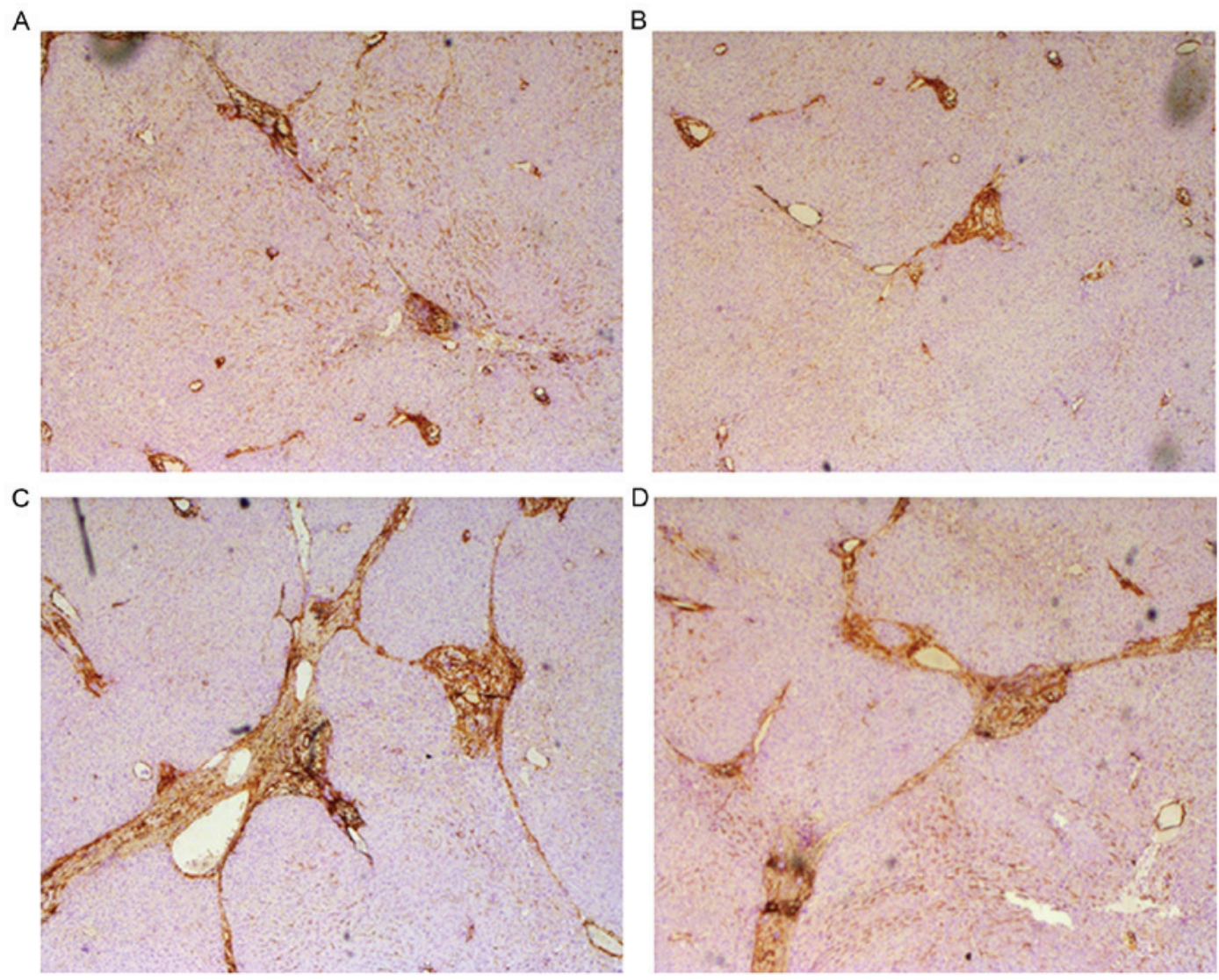

Figure 5. Effects of BM-MSC and AD-MSC transplantation on $\mathrm{CCl}_{4}$-induced liver fibrosis in rats. Immunocytochemical staining of liver fibrosis markers was performed and brown staining indicated fibrotic protein expression in the liver tissue. Representative images depicting morphological changes in rat liver tissues of the following groups are demonstrated: (A) AD-MSC; (B) BM-MSC; (C) negative and (D) blank control groups (magnification, x100). BM-MSCs, bone marrow-derived mesenchymal stem cells; AD-MSCs, adipose tissue-derived mesenchymal stem cells.

and IL-10 levels between the two groups. It has been widely reported that these cytokines are involved in regulating the proliferation, activation and apoptosis of HSCs; therefore, we postulated that the distinct effects of BM-MSCs and AD-MSCs on HSCs may be attributed to the differential levels of these cytokines released by BM-MSCs and AD-MSCs. IL-10 is a well-known anti-inflammatory cytokine capable of inhibiting the synthesis of pro-inflammatory cytokines, blocking the activity of nuclear factor- $\kappa \mathrm{B}$, enhancing $\mathrm{B}$-cell proliferation and affecting the cytokine-activated Janus kinase-signal transducer and activator of transcription signaling pathway (31). It was demonstrated in the present study that the secretion of IL-10 into the media of each MSC type did not differ significantly, which may explain, at least in part, why the effects of BM-MSCs and AD-MSCs on HSC activation are very similar.

The present study also evaluated the effectiveness of BM-MSCs and AD-MSCs in the treatment of liver fibrosis using a rat model of liver fibrosis, which was induced by injection of $\mathrm{CCl}_{4}$. The $\mathrm{CCl}_{4}$-induced animal model has a number of benefits as a suitable animal model for studying liver fibrosis with patterns similar to those of liver fibrotic disease in humans (32). However, the $\mathrm{CCl}_{4}$-induced animal model has a number of limitations (32), as the model cannot replicate the exact condition of liver fibrosis in humans and cannot represent distinctions in immune responses, gene expression and regulation. This limits the conclusions that can be drawn from the present study. The current study also investigated an animal model of liver fibrosis. It was demonstrated that inflammatory activity and fibrosis staging scores were significantly lower in MSC-treated groups than in the control groups. Furthermore, implantation of AD-MSCs slightly improved the anti-inflammatory and anti-fibrotic effects compared with BM-MSCs. However, these differences were not significant, which is inconsistent with the in vitro observations of the present study. In vitro studies investigating the effectiveness of AD-MSCs and BM-MSCs may lead to results that do not correspond with the results from animal models. Furthermore, the possibility that a range of immune responses in the animal model are involved could not be excluded, since animal models are far more complex than the co-culture system. Further investigations are currently under way in our laboratory to improve understanding of the underlying cellular and molecular mechanisms responsible for the effects of BM-MSCs and AD-MSCs, and to advance knowledge regarding the ability of BM-MSCs and AD-MSCs to combat liver fibrosis.

In conclusion, the results of the present study indicate that BM-MSCs and AD-MSCs are similarly effective at attenuating liver fibrosis by inhibiting the activation and proliferation of HSCs, as well as promoting the apoptosis of HSCs. Considering that AD-MSCs are easier to prepare and more effective at inhibiting HSC proliferation and apoptosis in the co-culture system used in the present study, and that the implantation of AD-MSCs exhibited slightly improved anti-inflammatory and anti-liver fibrotic activities compared 
with $\mathrm{BM}-\mathrm{MSCs}$ in the rat model of $\mathrm{CCl}_{4}$-induced liver fibrosis, AD-MSCs may be a better candidate than other MSCs for cell-based therapy to treat liver fibrosis. Future clinical studies and an understanding of the therapeutic mechanisms are required to advance understanding of the effects of AD-MSCs and BM-MSCs in the treatment of liver fibrosis.

\section{References}

1. Friedman SL: Liver fibrosis-from bench to bedside. J Hepatol 38 (Suppl 1): S38-S53, 2003.

2. Bosch J and García-Pagán JC: Complications of cirrhosis I. Portal hypertension. J Hepatol 32 (1 Suppl): S141-S156, 2000.

3. Cárdenas A: Hepatorenal syndrome: A dreaded complication of end-stage liver disease. Am J Gastroenterol 100: 460-467, 2005.

4. Bataller R and Brenner DA: Liver fibrosis. J Clin Invest 115: 209-218, 2005

5. Friedman SL: Hepatic stellate cells: Protean, multifunctional, and enigmatic cells of the liver. Physiol Rev 88: 125-172, 2008.

6. Salem HK and Thiemermann C: Mesenchymal stromal cells: Current understanding and clinical status. Stem Cells 28: 585-596, 2010.

7. Dezawa M, Ishikawa H, Itokazu Y, Yoshihara T, Hoshino M, Takeda S, Ide C and Nabeshima Y: Bone marrow stromal cells generate muscle cells and repair muscle degeneration. Science 309: 314-317, 2005.

8. Das M, Sundell IB and Koka PS: Adult mesenchymal stem cells and their potency in the cell-based therapy. J Stem Cells 8: 1-16, 2013.

9. Lu T, Yang C, Sun H, Lv J, Zhang F and Dong XJ: FGF4 and HGF promote differentiation of mouse bone marrow mesenchymal stem cells into hepatocytes via the MAPK pathway. Genet Mol Res 13: 415-424, 2014.

10. Ren G, Chen X, Dong F, Li W, Ren X, Zhang Y and Shi Y: Concise review: Mesenchymal stem cells and translational medicine: Emerging issues. Stem Cells Transl Med 1: 51-58, 2012

11. Sakaida I, Terai S, Yamamoto N, Aoyama K, Ishikawa T, Nishina $\mathrm{H}$ and Okita K: Transplantation of bone marrow cells reduces $\mathrm{CCl} 4$-induced liver fibrosis in mice. Hepatology 40 1304-1311, 2004.

12. Chamberlain G, Fox J, Ashton B and Middleton J: Concise review: Mesenchymal stem cells: Their phenotype, differentiation capacity, immunological features, and potential for homing. Stem Cells 25: 2739-2749, 2007.

13. Qi Z, Zhang Y, Liu L, Guo X, Qin J and Cui G: Mesenchymal stem cells derived from different origins have unique sensitivities to different chemotherapeutic agents. Cell Biol Int 36: 857-862, 2012.

14. Berardis S, Lombard C, Evraerts J, El Taghdouini A, Rosseels V, Sancho-Bru P, Lozano JJ, van Grunsven L, Sokal E and Najimi M: Gene expression profiling and secretome analysis differentiate adult-derived human liver stem/progenitor cells and human hepatic stellate cells. PLoS One 9: e86137, 2014.

15. Takeda M, Yamamoto M, Isoda K, Higashiyama S, Hirose M, Ohgushi H, Kawase M and Yagi K: Availability of bone marrow stromal cells in three-dimensional coculture with hepatocytes and transplantation into liver-damaged mice. J Biosci Bioeng 100: $77-81,2005$

16. Yu F, Ji S, Su L, Wan L, Zhang S, Dai C, Wang Y, Fu J and Zhang Q: Adipose-derived mesenchymal stem cells inhibit activation of hepatic stellate cells in vitro and ameliorate rat liver fibrosis in vivo. J Formos Med Assoc 114: 130-138, 2015.

17. Liu Y, Wang Z, Wang J, Lam W, Kwong S, Li F, Friedman SL, Zhou S, Ren Q, Xu Z, et al: A histone deacetylase inhibitor, largazole, decreases liver fibrosis and angiogenesis by inhibiting transforming growth factor- $\beta$ and vascular endothelial growth factor signalling. Liver Int 33: 504-515, 2013.
18. Majumder S, Piguet AC, Dufour JF and Chatterjee S: Study of the cellular mechanism of Sunitinib mediated inactivation of activated hepatic stellate cells and its implications in angiogenesis. Eur J Pharmacol 705: 86-95, 2013.

19. Chen YX, Huang YH, Zheng WD, Chen ZX, Zhang LJ and Wang XZ: Interleukin-10 gene modification attenuates hepatocyte activation of rat hepatic stellate cells in vitro. Mol Med Rep 7: 371-378, 2013.

20. Yu F, Su L, Ji S, Zhang S, Yu P, Zheng Y and Zhang Q: Inhibition of hepatic stellate cell activation and liver fibrosis by fat-specific protein 27. Mol Cell Biochem 369: 35-43, 2012.

21. Sumida Y, Nakajima A and Itoh Y: Limitations of liver biopsy and non-invasive diagnostic tests for the diagnosis of nonalcoholic fatty liver disease/nonalcoholic steatohepatitis. World J Gastroenterol 20: 475-485, 2014.

22. Yu J,Zhang S, Chu ES, Go MY, Lau RH, Zhao J, Wu CW, Tong L, Zhao J, vPoon TC and Sung JJ: Peroxisome proliferator-activated receptors gamma reverses hepatic nutritional fibrosis in mice and suppresses activation of hepatic stellate cells in vitro. Int J Biochem Cell Biol 42: 948-957, 2010.

23. Liu Y, Wang Z, Wang J, Lam W, Kwong S, Li F, Friedman SL, Zhou S, Ren Q, Xu Z, et al: A histone deacetylase inhibitor, largazole, decreases liver fibrosis and angiogenesis by inhibiting transforming growth factor- $\beta$ and vascular endothelial growth factor signaling. Liver Int 33: 504-515, 2013.

24. Huang X, Wang X, Lv Y, Xu L, Lin J and Diao Y: Protection effect of kallistatin on carbon tetrachloride-induced liver fibrosis in rats via antioxidative stress. PLoS One 9: e88498, 2014.

25. Sato Y, Araki H, Kato J, Nakamura K, Kawano Y, Kobune M, Sato T, Miyanishi K, Takayama T, Takahashi M, et al: Human mesenchymal stem cells xenografted directly to rat liver are differentiated into human hepatocytes without fusion. Blood 106: 756-763, 2005

26. Sharma RR, Pollock K, Hubel A and McKenna D: Mesenchymal stem or stromal cells: A review of clinical applications and manufacturing practices. Transfusion 54: 1418-1437, 2014.

27. Wang J, Bian C, Liao L, Zhu Y, Li J, Zeng L and Zhao RC: Inhibition of hepatic stellate cells proliferation by mesenchymal stem cells and the possible mechanisms. Hepatol Res 39: 1219-1228, 2009.

28. Haddad R and Saldanha-Araujo F: Mechanisms of T-cell immunosuppression by mesenchymal stromal cells: What do we know so far? Biomed Res Int 2014: 216806, 2014.

29. Sakaida I, Terai S, Yamamoto N, Aoyama K, Ishikawa T, Nishina $\mathrm{H}$ and Okita K: Transplantation of bone marrow cells reduces CCl4-induced liver fibrosis in mice. Hepatology 40: 1304-1311, 2004.

30. Ma ZG, Lv XD, Zhan LL, Chen L, Zou QY, Xiang JQ, Qin JL, Zhang WW, Zeng ZJ, Jin H, et al: Human urokinase-type plasminogen activator gene-modified bone marrow-derived mesenchymalstem cells attenuate liver fibrosis in rats by down-regulating the Wnt signaling pathway. World J Gastroenterol 22: 2092-2103, 2016.

31. Iyer SS and Cheng G: Role of interleukin 10 transcriptional regulation in inflammation and autoimmune disease. Crit Rev Immunol 32: 23-63, 2012.

32. Liedtke C, Luedde T, Sauerbruch T, Scholten D, Streetz K, Tacke F, Tolba R, Trautwein C, Trebicka J and Weiskirchen R: Experimental liver fibrosis research: Update on animal models, legal issues and translational aspects. Fibrogenesis Tissue Repair 6: 19, 2013. 